\title{
Tendencias y transformaciones educativas al integrar tecnologías en Educación Superior: el caso de la revista Uni-pluriversidad
}

\author{
Educational Trends and Transformations when Integrating Technologies in
} Higher Education: The Case of the Uni-pluriversidad Journal

\section{Jaime Andrés Carmona-Mesa* (D) Mónica Eliana Cardona Zapata** (D) Vanessa Arias Gil ${ }^{* *}$ iD}

\section{Tipo de artículo:}

informes de investigación y ensayos inéditos. Separata: Uni-pluriversidad 20 años.

Doi: 10.17533/udea.unipluri.20.3.01

\section{Cómo citar este artículo:}

Carmona-Mesa, J.A., Cardona Zapata, M.E, y Arias Gil, V (2020). Tendencias y transformaciones educativas al integrar tecnologías en Educación Superior: el caso de la revista Uni-pluriversidad, Uni-pluriversidad, 20(3), 1-17. https://doi. org/10.17533/udea.unipluri.20.3.01

Recibido: 13-09-2020 / Aprobado: 28-12-2020

\section{Resumen}

El crecimiento acelerado de la incorporación de tecnologías en los procesos educativos evidencia la necesidad de reconocer su impacto en los diferentes niveles de formación y particularmente en la educación superior. Este estudio tiene por objetivo identificar las tendencias y transformaciones educativas registradas en las investigaciones que hacen uso de tecnologías en la Educación Superior y que fueron reportadas en la revista Uni-pluriversidad entre el 2010 y 2020. Para ello, se recurre al análisis documental, dentro del cual se consideraron como núcleos temáticos los tipos de tecnologías implementadas y su uso como objetos de estudio o como medios para la transformación de procesos educativos. Como resultados principales se reporta la influencia y proyección de la revista a nivel de América Latina, un interés creciente por las discusiones que vinculan tecnologías y políticas públicas, una predominancia de trabajos con tecnologías digitales y un énfasis en la transformación de procesos en la formación de profesores a partir del uso de tecnologías. Finalmente, se plantean como oportunidades para la investigación en esta línea, el trabajo a partir de diferentes paradigmas de investigación que permitan ampliar la comprensión sobre las potencialidades y limitaciones de estas tecnologías en la Educación Superior, así como la necesidad de integrar diferentes disciplinas en este proceso.

Palabras clave: tecnología, educación superior, transformación educativa.

\footnotetext{
* Facultad de Educación-Universidad de Antioquia.

E-mail: jandres.carmona@udea.edu.co

** Facultad de Educación-Universidad de Antioquia.

E-mail: meliana.cardona@udea.edu.co

*** Facultad de Educación-Universidad de Antioquia. E-mail: vanessa.arias@udea.edu.co
}

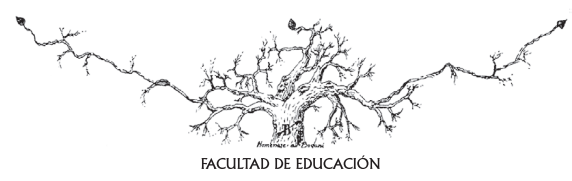




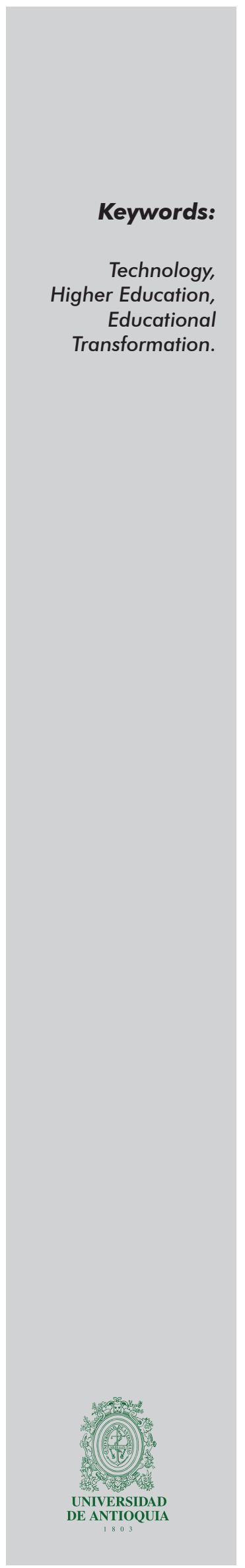

\section{Abstract}

An increased incorporation of new technologies in educational process demonstrates the need to recognize its impact on different levels of training and particularly on Higher Education. This study aims to identify educational trends and transformations which were reported in research that make use of technologies in Higher Education and published in the Uni-pluriversidad Journal between 2010 and 2020. For this purpose, documentary analysis is used to consider, as thematic nuclei, types of technologies implemented and their use as objects of study or as means for the transformation of educational processes. Main results indicate the influence and projection of the Journal at the Latin America context, a growing interest in discussions about technologies and public policies, a predominance of works with digital technologies, and an emphasis on the transformation of processes in teacher training by using technologies. Finally, some opportunities for research in this line are presented: working according to different research paradigms that allow for a broader understanding of the potential and limitations of these technologies in Higher Education, and the need to integrate different disciplines in this process. 
Introducción

La tecnología se ha constituido como un factor que impulsa cambios profundos en la sociedad y especialmente en educación a causa de los desafíos que demanda en los modos y patrones de relacionamiento entre los sujetos, el conocimiento y la infraestructura; lo anterior ha motivado diversidad de estudios que atienden a desafíos particulares sobre su implementación e impacto en la educación. Por ejemplo, Quiroga-Parra et al. (2017) realizan un estudio centrado en la capacidad tecnológica instalada en América Latina $y$, entre sus conclusiones, informan que el uso de la internet y de computadores es relativamente bajo en la región; es decir, el relacionamiento entre los sujetos y la infraestructura es limitada y, por ende, el dinamismo productivo, económico y social no se desarrolla con el potencial suficiente.

Por otra parte, investigaciones como la de Ávila-Fajardo y Riascos-Erazo (2011) han centrado la atención en la Educación Superior como referente para atender los desafíos demandados por estos nuevos modos de relacionamiento. Estos investigadores analizan diferentes modelos pedagógicos que les permiten establecer una propuesta propia para medir el impacto de las tecnologías en la enseñanza universitaria. Entre sus conclusiones, los autores plantean que la incursión de tecnologías en los ambientes académicos es un proceso que requiere una constante evaluación del impacto de estas herramientas, para optimizar su inclusión. En esa línea es posible inferir que, además de disponer de inversión para potenciar la infraestructura tecnológica, en el contexto educativo es necesa- rio ampliar la comprensión del relacionamiento entre los sujetos y el conocimiento cuando las herramientas tecnológicas tienen intermediación.

La preocupación por los cambios que genera la tecnología en la Educación Superior se ratifica en diversos estudios. Al respecto, Jensen (2019) reporta que, si bien las instituciones de Educación Superior difieren en su naturaleza, alcances y contextos, los avances tecnológicos presentan como desafío común la cuestión de cómo adaptarse y dar forma a la educación en un mundo digital. El autor complementa que la tecnología en sí misma es un medio para un fin; por lo tanto, es esencial debatir, cuestionar e indagar sobre las tendencias y transformaciones educativas que son reportadas en la comunidad académica.

En coherencia con las inquietudes reportadas por Jensen (2019) es importante ampliar en estudios que aporten evidencia de los alcances y limitaciones de las diferentes tecnologías que son integradas en la Educación Superior; información que funja como insumo en la toma de decisiones para su inclusión (Ávila-Fajardo y Riascos-Erazo, 2011). En ese sentido, este artículo tiene como propósito desarrollar un análisis de las tendencias y transformaciones educativas al integrar tecnologías en la Educación Superior que han sido reportadas en la revista Uni-pluriversidad. Por lo tanto, se describe a continuación la metodología, seguida de los principales resultados del estudio y las conclusiones que permiten informar los aportes de la revista a la comunidad académica y la región.

\section{Metodología}

Para reconocer la producción académica de la revista Uni-pluriversidad en relación con la integración de tecnologías en la Educación Superi- or, nivel de formación declarado como enfoque de la revista, se tuvieron en cuenta algunos elementos propuestos por Hoyos (2000) sobre la metod- 
ología de investigación documental. En especial, se retoma el concepto de núcleos temáticos (subtemas que delimitan los objetos de conocimiento), unidades de análisis (cada uno de los textos que configuran el acervo documental a estudiar), factores (elementos que se distinguen como relevantes en las unidades de análisis y que contribuyen a un análisis por núcleos temáticos); así como el conjunto de estrategias propuestas por la autora para el procedimiento de análisis y la construcción teórica global.

De acuerdo con lo anterior y considerando las tendencias reconocidas en otras investigaciones con la misma naturaleza y especificidad (p. ej. Marín et al., 2018), en la Tabla 1 se presentan los núcleos temáticos y los factores definidos para el análisis de cada unidad.

Tabla 1. Núcleos temáticos y factores para el análisis

\begin{tabular}{ll}
\hline Núcleos temáticos & Factores \\
\hline Tipos de tecnologías implementadas & Población o público objetivo \\
\hline Usos de la tecnología & Tipo de investigación \\
& Conceptos o temáticas \\
& Referentes teóricos y metodológicos \\
\hline
\end{tabular}

En coherencia con los núcleos delimitados en Tabla 1, la tecnología se asume bajo una visión integradora de análogas y digitales, que trasciende la concepción de recursos aislados de un contexto o una praxis y que, a partir de su uso, transforma lo que es cognoscible y la manera en que algo puede conocerse en el proceso educativo
(Carmona-Mesa, Salazar y Villa-Ochoa, 2018). Así mismo, los factores cobran importancia en el proceso de análisis de las unidades seleccionadas al destacar las particularidades contextuales y epistemológicas de las investigaciones, lo cual permitió fortalecer las inferencias generadas en relación con los núcleos temáticos delimitados.

Tabla 2. Unidades de análisis

\begin{tabular}{cccl}
\hline Volumen & Número & $\begin{array}{c}\text { Cantidad de } \\
\text { artículos }\end{array}$ & Autores \\
\hline 11 & 1 & 2 & Ramírez (2011); Vargas (2011) \\
\cline { 2 - 5 } & 3 & 1 & Ospina (2011) \\
\hline 12 & 1 & 1 & Zapata, Marín y Vélez (2012) \\
\hline 13 & 2 & 1 & Bolívar (2013) \\
\cline { 2 - 5 } & 3 & 1 & Medina y Ortiz (2013) \\
\hline 14 & 2 & 3 & Vásquez (2014); Martins (2014); Hugo et al. (2014) \\
\hline 16 & 1 & 1 & Richit (2014) \\
\hline 17 & 1 & 1 & Martínez, Susano y Espinosa (2015) \\
\hline 18 & 1 & 1 & Rueda y Parada (2016) \\
\hline 19 & 2 & 3 & $\begin{array}{l}\text { Fabro (2017); Muchenski, Miquelin y da Silva (2017); Carrillo } \\
\text { y Suárez (2017) }\end{array}$ \\
\hline & 1 & 5 & $\begin{array}{l}\text { Carmona-Mesa, Salazar y Villa-Ochoa (2018) } \\
\text { Aró, Villafañe y Degrave (2020); Zampieri y Javaroni (2020); (2019); Rosa y Orey (2019); Romo-Vázquez, } \\
\text { Vera, Araujo y Veit (2020); Quiroz-Vallejo (2020) }\end{array}$ \\
\hline
\end{tabular}


En particular, se consultaron todos los números publicados por la revista Uni-pluriversidad (volúmenes 10 a 20) entre los años 2010 y 2020, con un total de 300 artículos, de los cuales se seleccionaron 34 como unidades de análisis, considerando que abordaban el concepto de tecnología en diferentes áreas de conocimiento. Se establecieron como criterios de selección que fueran trabajos de investigación, reflexiones teóricas y entrevistas que hicieran referencia a tecnologías análogas o digitales como objetos de estudio, o como medios utilizados en la transformación de procesos de formación.

Así mismo, se consideran criterios de exclusión: 1) las secciones de editorial, 2) revisión de libros y/o novedades bibliográficas, 3) aquellos estudios que hacían referencia a la tecnología en sentido amplio o presentaban ideas a partir de una perspectiva antropológica del concepto, no relacionada con el ámbito educativo y 4) los que estaban dirigidos a niveles de formación diferentes a la Educación Superior. Al tener en consideración estos criterios, el número de unidades de análisis seleccionados es 24 (Tabla 2).

Para el proceso de análisis y la construcción teórica, siguiendo los planteamientos de Hoyos (2000), cada uno de los investigadores realizó un proceso de interpretación de las unidades seleccionadas por cada núcleo temático para identificar los factores asociados y presentar las tendencias en cuanto al uso de tecnologías en educación superior y las transformaciones educativas reportadas por los autores presentados en la Tabla 2. Posteriormente, se realizó un proceso de triangulación entre investigadores y con los referentes teóricos para consolidar el corpus teórico que da lugar a los resultados y su discusión.

\section{Resulfados}

Los resultados de la presente revisión muestran algunas tendencias en la implementación de tecnologías para diferentes procesos educativos, identificadas en artículos de investigación y de reflexión que fueron desarrollados principalmente por investigadores de universidades públicas y privadas en países como Colombia, Brasil, Argentina, Venezuela y México; España y Portugal, en menor medida (Tabla 3). El origen geográfico de estos estudios da cuenta de que, si bien Uni-pluriversidad tiene un alcance en el objeto de interés a nivel iberoamericano, se considera como una opción para la publicación principalmente por parte de investigadores de América Latina.

La ubicación geográfica de las investigaciones reportadas, además de brindar información del reconocimiento de la revista Uni-pluriversidad a nivel Iberoamericano, también denota los alcances de las posibles inferencias derivadas de los núcleos temáticos (tipos de tecnologías implementadas y usos de la tecnología). En otras palabras, las discusiones y evidencia empírica del tipo y uso de tecnología limitan las argumentos y conclusiones de este estudio principalmente al ámbito de América Latina.

En relación con las metodologías de investigación, 18 artículos tienen metodología de investigación cualitativa, uno investigación cuantitativa, uno metodología de investigación mixta, dos reflexión y dos entrevistas. En la Tabla 4 se muestran los principales factores que permiten la caracterización general de los trabajos revisados. Entre estos factores se resalta que la mitad de los trabajos están orientados hacia los profesores en formación (11) o en ejercicio (2) y los demás están distribuidos entre estudiantes de carreras como ingeniería (1), administración (1), diseño (1), economía (1), investigación (2), estudiantes sordos (1), bioquímica (1) y comunidad en general (3). Con respecto a los conceptos clave, se encontraron trabajos en los que predomina la integración de tecnologías en la formación, la inclusión y alfabetización digital, la educación en Ciencia, Tecnología y Sociedad (CTS), la modelación matemática, entre otras. 
Tabla 3. Universidades y países en los que se han realizado los estudios

\begin{tabular}{|c|c|c|c|}
\hline País & Universidades & $\begin{array}{c}\text { Cantidad } \\
\text { de artículos }\end{array}$ & Autores \\
\hline \multirow[t]{4}{*}{ Colombia } & Universidad de Antioquia & 7 & $\begin{array}{l}\text { Ramírez (2011); Ospina (2011); Zapata, } \\
\text { Marín y Vélez (2012); Bolívar (2013); Car- } \\
\text { mona-Mesa, Salazar y Villa-Ochoa (2018); } \\
\text { Carmona-Mesa, Cardona y Castrillón-Yepes } \\
\text { (2020); Quiroz-Vallejo (2020) }\end{array}$ \\
\hline & Universidad de los Andes & 1 & Velasco y Gómez (2019) \\
\hline & $\begin{array}{l}\text { Universidad Industrial de } \\
\text { Santander }\end{array}$ & 1 & Rueda y Parada (2016) \\
\hline & $\begin{array}{l}\text { Universidad Minuto de } \\
\text { Dios y Universidad Santo } \\
\text { Tomás }\end{array}$ & 1 & Carrillo y Suárez (2017) \\
\hline \multirow[t]{4}{*}{ Brasil } & $\begin{array}{l}\text { Universidade Federal da } \\
\text { Fronteira Sul (UFFS) }\end{array}$ & 1 & Richit (2014) \\
\hline & $\begin{array}{l}\text { Universidade Tecnológica } \\
\text { Federal do Paraná / Colé- } \\
\text { gio Estadual do Paraná }\end{array}$ & 1 & Muchenski, Miquelin y da Silva (2017) \\
\hline & $\begin{array}{l}\text { Univer- sidade Federal de } \\
\text { Ouro Preto (UFOP), }\end{array}$ & 1 & Rosa y Orey (2019) \\
\hline & $\begin{array}{l}\text { Universidad Es- tatal de } \\
\text { Sao Paulo (UNESP), Rio } \\
\text { Claro (Sao Paulo) }\end{array}$ & 1 & Zampieri y Javaroni (2020) \\
\hline \multirow[t]{2}{*}{$\begin{array}{l}\text { Colombia y } \\
\text { Brasil }\end{array}$} & $\begin{array}{l}\text { Colombia: Universidad } \\
\text { Distrital Francisco José } \\
\text { de Caldas y Universidad } \\
\text { de Antioquia. }\end{array}$ & & Vera, Araujo y Veit (2020) \\
\hline & $\begin{array}{l}\text { Brasil: Universidade Fe- } \\
\text { deral de Rio Grande do } \\
\text { Sul (UFRGS) e Instituto } \\
\text { Federal Sul-Rio-Granden- } \\
\text { se/Vis- } \\
\text { conde da Graça (IFSul) }\end{array}$ & 1 & \\
\hline \multirow[t]{2}{*}{ Argentina } & $\begin{array}{l}\text { Universidad Nacional del } \\
\text { Comahue }\end{array}$ & 1 & Hugo et al. (2014) \\
\hline & $\begin{array}{l}\text { Universidade Nacional do } \\
\text { Litoral }\end{array}$ & 2 & $\begin{array}{l}\text { Fabro (2017) } \\
\text { Fabro et al. (2020) }\end{array}$ \\
\hline \multirow[t]{2}{*}{ España } & Universidad de Granada & 1 & Vargas (2011) \\
\hline & $\begin{array}{l}\text { Universidad de las Islas } \\
\text { Baleares }\end{array}$ & 1 & Vásquez (2014) \\
\hline \multirow[t]{2}{*}{ México } & $\begin{array}{l}\text { Universidad Autónoma } \\
\text { de San Luis Potosí }\end{array}$ & 1 & Martínez, Susano y Espinosa (2015) \\
\hline & $\begin{array}{l}\text { Centro de Investigación } \\
\text { en Ciencia Aplicada y } \\
\text { Tecnología Avanzada de } \\
\text { México }\end{array}$ & 1 & Romo-Vázquez, Barquero y Bosch (2019) \\
\hline Venezuela & Universidad de Carabobo & 1 & Medina y Ortiz (2013) \\
\hline Portugal & Universidade de Aveiro & 1 & Martins (2014) \\
\hline
\end{tabular}


Tabla 4. Factores para la caracterización de las unidades de análisis

\begin{tabular}{|c|c|c|}
\hline Unidad de análisis & $\begin{array}{l}\text { Factor 1: Población o público obje- } \\
\text { tivo }\end{array}$ & Factor 2: Conceptos clave \\
\hline Ramírez (2011) & Profesores e ingenieros en formación & $\begin{array}{l}\text { Integración de las TIC en pro- } \\
\text { cesos educativos }\end{array}$ \\
\hline Vargas (2011) & Administradores en formación & Administración pública \\
\hline $\begin{array}{l}\text { Zapata, Marín y Vélez } \\
(2012)\end{array}$ & Diseñadores en formación & Funciones \\
\hline Ospina (2011) & Investigadores & Identidad, cultura \\
\hline Medina y Ortiz (2013) & $\begin{array}{l}\text { Estudiantes de un curso de introduc- } \\
\text { ción a la matemática de la Facultad } \\
\text { de Ciencias Económicas }\end{array}$ & Modelización matemática \\
\hline Bolívar (2013) & Profesores en formación & Uso de TIC \\
\hline Richit (2014) & Profesores en formación & Inclusión digital \\
\hline Vásquez (2014) & Profesores en formación & $\begin{array}{l}\text { Enseñar, aprender y evaluar } \\
\text { Educación CTS }\end{array}$ \\
\hline Martins (2014) & Profesores en formación & $\begin{array}{l}\text { Políticas públicas } \\
\text { Educación CTS }\end{array}$ \\
\hline $\begin{array}{l}\text { Hugo, Olavegogeasoechea, } \\
\text { Salica, Orlandini y Ávila } \\
\text { (2014) }\end{array}$ & Profesores en formación & $\begin{array}{l}\text { Naturaleza de la ciencia y tec- } \\
\text { nología (NdCyT) } \\
\text { Energía } \\
\text { Educación CTS }\end{array}$ \\
\hline $\begin{array}{l}\text { Martínez, Susano y Espi- } \\
\text { nosa (2015) }\end{array}$ & $\begin{array}{l}\text { Materias de metodología de la inves- } \\
\text { tigación, psicología industrial y neu- } \\
\text { romarketing }\end{array}$ & Concrevirtualidad \\
\hline Rueda y Parada (2016) & Estudiantes de Ingeniería & Razonamiento covariacional \\
\hline Fabro (2017) & Estudiantes de Bioquímica & $\begin{array}{l}\text { Ciencias morfológicas } \\
\text { B-learning }\end{array}$ \\
\hline $\begin{array}{l}\text { Muchenski, Miquelin y da } \\
\text { Silva (2017) }\end{array}$ & Comunidad académica en general & Inclusión digital \\
\hline Carrillo y Suárez (2017) & Estudiantes sordos universitarios & $\begin{array}{l}\text { Diseño de actividades didácticas } \\
\text { para el uso de la lengua escrita }\end{array}$ \\
\hline $\begin{array}{l}\text { Carmona-Mesa, Salazar y } \\
\text { Villa-Ochoa (2018) }\end{array}$ & $\begin{array}{l}\text { Formación inicial de profesores de } \\
\text { Matemáticas }\end{array}$ & $\begin{array}{l}\text { Diseño de cursos específicos de } \\
\text { FIP }\end{array}$ \\
\hline Velasco y Gómez (2019) & No aplica & MOOC \\
\hline Rosa y Orey (2019) & $\begin{array}{l}\text { Formación inicial de profesores de } \\
\text { Matemáticas }\end{array}$ & Modelación Matemática \\
\hline $\begin{array}{l}\text { Romo-Vázquez, Barquero y } \\
\text { Bosch (2019) }\end{array}$ & Formación de profesores & Modelización matemática. \\
\hline $\begin{array}{l}\text { Carmona-Mesa, Cardona y } \\
\text { Castrillón-Yepes (2020) }\end{array}$ & $\begin{array}{l}\text { Formación inicial de profesores de } \\
\text { matemáticas }\end{array}$ & $\begin{array}{l}\text { Integra ciencias, tecnología y } \\
\text { matemáticas a través del estudio } \\
\text { del calentamiento del agua. } \\
\text { Educación STEM }\end{array}$ \\
\hline $\begin{array}{l}\text { Fabro, Aró, Villafañe y De- } \\
\text { grave (2020) }\end{array}$ & Educación superior en general & $\begin{array}{l}\text { Ciencias Morfológicas } \\
\text { B-learning }\end{array}$ \\
\hline Zampieri y Javaroni (2020) & Educación continua de profesores & Pensamiento computacional \\
\hline Vera, Araujo y Veit (2020) & Profesores de ciencias en formación & $\begin{array}{l}\text { CoP virtual / Curso virtual de ex- } \\
\text { tensión sobre Recursos Educati- } \\
\text { vos Abiertos (REA) }\end{array}$ \\
\hline Quiroz-Vallejo (2020) & Profesores en general & Pensamiento computacional \\
\hline
\end{tabular}


En particular, los trabajos de Bolívar (2013) y Quiroz-Vallejo (2020) presentan las transcripciones y discusiones de dos entrevistas realizadas a expertos en el uso de tecnologías para la educación, como el Dr. Octavio Henao Álvarez y el Dr. Johannes Krugel, respectivamente. Bolívar (2013), en su interlocución con el Dr. Henao, plantea algunas reflexiones en relación con el papel de la tecnología en la didáctica y en la formación de profesores; resalta que es necesaria la apropiación inteligente de la tecnología para asumir una postura crítica frente al desarrollo vertiginoso de la sociedad actual, estableciendo una relación fundamentada en el humanismo digital y manteniendo el rol activo de los profesores en los procesos de enseñanza. Así mismo, el Dr. Henao señala que las tecnologías por sí mismas no cualifican la educación, es decir:

Estas herramientas pueden ser extensiones interesantes de la mente, pero son recursos que solo cobran sentido en el marco de propuestas didácticas y enfoques pedagógicos adecuados. Sin un conocimiento disciplinar riguroso es imposible hacer una buena propuesta didáctica mediada por estas tecnologías. (Bolívar, 2013, p. 118)

Por su parte, Quiroz-Vallejo (2020) informa algunas implicaciones prácticas para la investigación a futuro en el Pensamiento Computacional (PC) para el caso de Colombia: por un lado, los desafíos en la formación de profesores y, por el otro, la tendencia en el tipo de actividades. Tanto la entrevista al Dr. Henao como la del Dr. Krugel llaman la atención por cierta correspondencia con los factores predominantes en la revista; en especial, existe una marcada relación entre los trabajos publicados a la fecha y las afirmaciones del Dr. Henao (ver Tabla 4). En ese sentido, es posible conjeturar que el PC se tornará como una temática de interés en las futuras publicaciones de la revista, lo cual conserva cohesión con las declaraciones de revisiones de literatura desarrolladas en la región y en el mismo nivel de formación (p. ej. Villa-Ochoa y Castrillón-Yepes, 2020).

Otro aspecto que se resalta en las unidades de análisis tiene relación con los trabajos de
Vásquez (2014), Martins (2014) y Hugo et al. (2014), quienes contemplan la perspectiva de la educación en Ciencia, Tecnología y Sociedad (CTS) con el propósito de discutir asuntos relacionados con la enseñanza, el aprendizaje, la evaluación, las políticas públicas, las directrices curriculares y la comprensión de la naturaleza de la Ciencia y la Tecnología. La perspectiva CTS se ha consolidado en la enseñanza de las ciencias por fomentar procesos metacognitivos, de reflexión y razonamiento, que son necesarios para el desarrollo de habilidades como la resolución de problemas, la creatividad y el pensamiento crítico (Vásquez, 2014).

De acuerdo con lo anterior, Hugo et al. (2014) plantean la necesidad de generar espacios en la formación de profesores de ciencias en los que se favorezca el desarrollo de estas habilidades en CTS, para que los futuros profesores adquieran las herramientas necesarias al orientar procesos para la alfabetización científico-tecnológica de todas las personas. Los anteriores planteamientos se pueden concretar en la propuesta presentada por Martins (2014), quien, a partir de un análisis realizado sobre las directrices curriculares en enseñanza de las ciencias y la legislación para la formación de profesores, concluye que las instituciones de formación de profesores deben establecer como prioridad la investigación en CTS, dado que esta contribuye a la mejora de la educación en general y al fortalecimiento de la investigación científica en educación.

Para las unidades restantes de análisis, se establecieron dos factores emergentes que permitieron identificar las tendencias en relación con la integración de tecnologías en Educación Superior y los diferentes usos dados a dichas tecnologías. Se distinguen aquellas en las que se centra la tecnología como objeto de estudio, y aquellas en las que se presenta la tecnología como medio para la transformación de procesos en diferentes áreas. Lo anterior, en coherencia con las tendencias reconocidas en otras investigaciones con la misma naturaleza y especificidad (Marín et al., 2018) y la visión de tecnología declarada en el marco metodológico (Carmona-Mesa, Salazar y Villa-Ochoa, 2018). A continuación, se discuten los principales hallazgos de estas tendencias. 


\section{Tendencias en los tipos de tecnologías implementadas en educación superior}

En esta primera categoría se describen los tipos de tecnologías (análogas o digitales) que se identificaron en 19 unidades de análisis (Tabla 5), a partir de las cuales se observó que pre- domina el uso de tecnologías digitales como la plataforma Moodle, las redes sociales, las aplicaciones para realidad virtual y aumentada y el uso de la internet en general.

Tabla 5. Tipos de tecnologías

\begin{tabular}{|c|c|c|c|}
\hline $\begin{array}{l}\text { Tipo de } \\
\text { tecnología }\end{array}$ & Herramienta & Autores & Total \\
\hline Digital & $\begin{array}{l}\text { Calculadora gráfica } \\
\text { CG Voyage } 200 \text { de Texas Instruments }\end{array}$ & Medina y Ortiz (2013) & 1 \\
\hline $\begin{array}{l}\text { Análoga y } \\
\text { Digital }\end{array}$ & Calculadoras simples y videojuegos & $\begin{array}{l}\text { Carmona-Mesa, Salazar y Vil- } \\
\text { la-Ochoa (2018) }\end{array}$ & 1 \\
\hline Digital & Plataforma Moodle & $\begin{array}{l}\text { Ramírez (2011); Fabro } \\
\text { (2017); Rosa y Orey (2019) }\end{array}$ & 3 \\
\hline Digital & Computadores (inclusión digital) & Richit (2014) & 1 \\
\hline Digital & MOOC & Velasco y Gómez (2019) & 1 \\
\hline Digital & GeoGebra & Rueda y Parada (2016) & 1 \\
\hline Digital & $\begin{array}{l}\text { Realidad virtual y aumentada, mod- } \\
\text { elos en 3D }\end{array}$ & $\begin{array}{l}\text { Zapata, Marín y Vélez (2012); } \\
\text { Fabro et al. (2020) }\end{array}$ & 2 \\
\hline Digital & Internet & $\begin{array}{l}\text { Vargas (2011); Ospina } \\
\text { (2011); Carrillo y Suárez } \\
\text { (2017) }\end{array}$ & 3 \\
\hline Digital & Redes sociales & $\begin{array}{l}\text { Martínez, Susano y Espino- } \\
\text { sa (2015); Romo-Vázquez, } \\
\text { Barquero y Bosch (2019) }\end{array}$ & 2 \\
\hline Digital & $\begin{array}{l}\text { Rocket.Chat / own- Cloud / OnlyOf- } \\
\text { fice }\end{array}$ & Vera, Araujo y Veit (2020) & 1 \\
\hline Digital & Scratch & Zampieri y Javaroni (2020) & 1 \\
\hline Digital & Smartphone & $\begin{array}{l}\text { Muchenski, Miquelin y da Sil- } \\
\text { va (2017) }\end{array}$ & 1 \\
\hline $\begin{array}{l}\text { Análoga y } \\
\text { Digital }\end{array}$ & $\begin{array}{l}\text { Termómetro de mercurio y simulación } \\
\text { computacional }\end{array}$ & $\begin{array}{l}\text { Carmona-Mesa, Cardona y } \\
\text { Castrillón-Yepes (2020) }\end{array}$ & 1 \\
\hline
\end{tabular}

Identificar los tipos de tecnología que se implementan es importante en cuanto informa sobre los posibles modos y patrones de relacionamiento entre los sujetos y la infraestructura priorizada en los estudios. Al respecto, si bien autores como Quiroga-Parra et al. (2017) informan que la capacidad tecnológica instalada en América Latina en cuanto a la internet y computadores es relativamente baja, la presente revisión da cuenta de un interés especial en tecnologías digitales so- portadas principalmente en la internet ( $p$. ej. En redes sociales y educativas). Además, se encuentra tecnologías vinculadas a computadores ( $p$. ej. GeoGebra y simulaciones computacionales) y, notoriamente en menor medida, los dispositivos móviles. Lo anterior contrasta parcialmente con el dinamismo productivo, económico y social actual, en donde la internet y los dispositivos móviles tienen un papel preponderante (Finquelievich, 2020). 
En relación con el uso de tecnologías como las calculadoras simples y graficadoras, se señalan los trabajos de Medina y Ortiz (2013) y Carmona-Mesa, Salazar y Villa-Ochoa (2018). En el primero, se realiza un análisis sobre el desarrollo de competencias matemáticas por parte de los estudiantes al hacer uso de calculadoras gráficas para apoyar procesos de modelización. En el segundo, los autores plantean la necesidad de fortalecer la formación de futuros profesores de matemáticas en relación con el uso de tecnologías, apoyándose en calculadoras simples y videojuegos para determinar los diferentes usos que pueden realizar los futuros profesores en la resolución de problemas y con proyección al ejercicio profesional.

En cuanto al uso de plataformas virtuales para el aprendizaje, tales como los cursos en Moodle, en trabajos como los de Ramírez (2011) y Fabro (2017) se identifica que el diseño de escenarios educativos apoyados en la plataforma Moodle favorece la interacción por medio de la hipertextualidad, los foros virtuales y la posibilidad de acceso a diferentes recursos educativos digitales que ayudan en la adquisición de conocimientos, el aprendizaje autónomo y la aplicación de conocimientos a diferentes situaciones. Por otra parte, Rosa y Orey (2019), proponen un Ambiente Virtual de Aprendizaje (AVA) para desarrollar proyectos de modelación matemática, con el apoyo de herramientas disponibles en Moodle; de acuerdo con estos autores, la metodología por proyectos permite que los estudiantes contextualicen sus problemas y el uso del AVA les ayuda en la gestión de la información necesaria para reflexionar de una manera crítica sobre el papel de las matemáticas en la cotidianidad.

En esta misma línea se encuentra el trabajo desarrollado por Velasco y Gómez (2019), quienes establecen algunos criterios que ayudan a identificar la calidad de los cuestionarios que se presentan en cursos abiertos y masivos en línea (MOOC), a partir de niveles de dificultad que permiten a los estudiantes poner en juego sus conocimientos. También se hallan los trabajos de Vargas (2011), Ospina (2011) y Carrillo y Suárez
(2017), quienes plantean diferentes propuestas en las que se destaca el papel de la internet para favorecer la interacción, el desarrollo de procesos a través de diferentes medios, la adquisición de competencias tecnológicas, la atención a la diversidad de estilos de aprendizaje y la gestión de grandes cantidades de información. No obstante, Ospina (2011) plantea una reflexión sobre la influencia de esta red de comunicación en la construcción de identidades, ya que su uso ha generado una transformación tanto a nivel educativo como político, al instaurar nuevos significados sobre las relaciones sociales, la vida cotidiana y la perspectiva sobre el mundo.

Con respecto a las relaciones sociales y a la posibilidad de generar vínculos entre diferentes comunidades, autores como Martínez, Susano y Espinosa (2015), Romo-Vázquez, Barquero y Bosch (2019) y Vera, Araujo y Veit (2020), coinciden en la necesidad de fortalecer la comunicación entre profesores por medio de diferentes canales como las redes sociales, las unidades de aprendizaje online y las Comunidades de Práctica (CoP) virtual. Estos canales fortalecen el desarrollo profesional, el acompañamiento pedagógico y didáctico en procesos académicos, el mejoramiento de habilidades tecnológicas para la implementación de diversas estrategias para la planeación, la enseñanza y la evaluación, el apoyo de colegas en diferentes niveles de formación y a la disminución de la brecha entre la investigación educativa y la práctica docente. No obstante, estos autores resaltan dificultades como la apropiación por parte de los profesores de diferentes plataformas virtuales por medio de los cuales se propician estos espacios. En relación con dicha apropiación, se considera importante que incluyan políticas que favorezcan la creación de espacios para la culturización digital, desde los niveles iniciales en los programas de formación de profesores, y que permanezcan de manera continua durante su ejercicio (Richit, 2014).

Por último, los trabajos de Rueda y Parada (2016), Zapata, Marín y Vélez (2012), Fabro et al. (2020), Zampieri y Javaroni (2020), 
Muchenski, Miquelin y da Silva (2017) y Carmona-Mesa, Cardona y Castrillón-Yepes (2020), hacen énfasis en tecnologías dirigidas hacia la integración en la enseñanza de diferentes áreas de conocimiento. Se pudo identificar que existen múltiples posibilidades para mejorar el apoyo a los procesos educativos, siempre y cuando se acompañen de estrategias didácticas que favorezcan el desarrollo de aprendizajes significativos, el trabajo colaborativo, el uso especializado de dispositivos como tablets y smartphones y la integración de diferentes disciplinas a partir de metodologías reconocidas con potencial en el marco de la educación STEM (Science, Technology, Engineering and Mathematics). De esta forma, se superan las barreras espaciotemporales para el acceso al conocimiento (Fabro et al., 2020) y se generan procesos colaborativos, en los que se unen esfuerzos para crear una cultura tecnológica que tenga un impacto en escenarios educativos, económicos, sociales y ambientales.

Identificar las tendencias en los usos de las tecnologías en la Educación Superior es importante en cuanto permite comprender los modos y patrones de relacionamiento entre los sujetos y la infraestructura delimitados a partir de los tipos de tecnología. Así mismo, le da un lugar protagónico al conocimiento en estos relacionamientos a partir de la evidencia empírica (Ávila-Fajardo y Riascos-Erazo, 2011). Al respecto, si bien se reconoce la diversidad latente entre las instituciones de Educación Superior (Jensen, 2019), se registra como desafío común la preocupación por explorar formas para adaptar la educación a las demandas sociales emergentes $y$, a pesar de considerar las reflexiones teóricas como criterio de inclusión, en la mayoría de los estudios se informan resultados favorables a partir de evidencia empírica.

No obstante, es importante ampliar las discusiones que permitan plantear rutas para la generalización de las reflexiones logradas de forma situada, es decir, trascender a orientaciones que permitan establecer rutas de trabajo en otros contextos y sin generar necesariamente investigaciones. Un ejemplo es el estudio de Velasco y Gómez (2019), quienes logran establecer criterios concretos para procurar mantener el mismo impacto que ellos logran, pero en futuros estudios.

\section{Tecnologías como objeto de estudio y medio para la transformación de procesos en Educación Superior}

Para esta categoría se consideraron las unidades de análisis que hacían referencia a dos subcategorías, si bien en algunos estudios se ubicaban en ambas categorías, esos estudios se organizaron de acuerdo con el principal uso iden- tificado. Al respecto, en la Tabla 6 se presentan los trabajos correspondientes a cada una de ellas, a saber: Tecnología como objeto de estudio y Transformación de procesos en educación superior.

Tabla 6. Uso dado a los tipos de tecnologías

\begin{tabular}{llc}
\hline \multicolumn{1}{c}{ Uso } & \multicolumn{1}{c}{ Autores } & Total \\
\hline $\begin{array}{ll}\text { Tecnología como } \\
\text { objeto de estudio }\end{array}$ & $\begin{array}{l}\text { Vélez (2012); Muchenski, Miquelin y da Silva (2017); Velasco y } \\
\text { Gómez (2019) }\end{array}$ & 6 \\
& & \\
\hline $\begin{array}{l}\text { Transformación } \\
\text { de procesos en }\end{array}$ & (2015); Rueda y Parada (2016); Fabro (2017); Carrillo y Suárez & 13 \\
educación supe- & (2017); Carmona-Mesa, Salazar y Villa-Ochoa (2018); Rosa y Orey & \\
rior & (2019); Romo-Vázquez, Barquero y Bosch (2019); Carmona-Mesa, & \\
& Cardona y Castrillón-Yepes (2020); Fabro et al. (2020); Vera, Araujo & \\
& y Veit (2020); Zampieri y Javaroni (2020) & \\
& &
\end{tabular}


Esta subcategoría refiere a la conceptualización y análisis de tecnologías a partir de su diseño, es decir, se presentan las investigaciones que discuten los alcances en términos de las nociones y desarrollos de software; se identifican seis documentos en esta especificidad (Tabla 6). Por ejemplo, Vargas (2011) desarrolla un estudio de revisión teórica que le permite delimitar los objetivos, funciones, ventajas, desventajas y dimensiones en torno a Gobierno Electrónico (e-Gobierno o e-Gob); por otro lado, Zapata, Marín y Vélez (2012) presentan una metodología para el diseño gráfico de entornos de enseñanza y aprendizaje en mundos virtuales tridimensionales.

En relación con la conceptualización de tecnología, Muchenski, Miquelin y da Silva (2017) estudian el reconocimiento y fomento de los teléfonos celulares en el aula a partir de documentos de disposiciones legales y organizaciones internacionales; se resalta que a pesar de reconocerse el desarrollo de esta tecnología, en ambos tipos de documentos existen divergencias. Los autores plantean la necesidad de establecer vínculos entre los gestores de políticas y los actores educativos que podrían aprovechar el desarrollo de los teléfonos. Por su parte, Ospina (2011) analiza los alcances de las redes telemáticas y sus diversos grados de inserción en la sociedad, así como las imágenes y conceptos que circulan de manera más rápida y masiva gracias a soportes como las pantallas accesibles; aspectos decisivos para la configuración de identidades digitales.

Por otro lado, en relación con la construcción de objetos de aprendizaje, Ramírez (2011) establece en su estudio una caracterización y contraste de una hipermedia con seis módulos (La Educación una aventura virtual) con un cam- pus virtual soportado en Moodle (Campus Virtual GITT). La autora reporta que la ruta analítica basada en algunos criterios de la metodología del aprender a aprender e inmersa en la línea de la comunicación educativa como eje transversal en la implementación de TIC, le permitió evaluar la naturaleza y calidad de ambos desarrollos tecnológicos en algunos factores fundamentales como la interacción, la retroalimentación y la mediación pedagógica.

De forma similar, Velasco y Gómez (2019) analizan el diseño de los cuestionarios de evaluación que se usan de forma habitual en los cursos abiertos, gratuitos, masivos en línea (MOOC por sus siglas en inglés), al resaltar la necesidad de evaluar la calidad relativa y la dificultad de los cuestionarios. En particular, estos investigadores indagan por un cuestionario considerado en el diseño de un curso de Educación Matemática para profesores de primaria (PriMat) y establecen que, si bien algunas preguntas se encuentran en los parámetros habituales, estas deben ser ampliadas o reducidas en la dificultad percibida por los participantes.

Este conjunto de hallazgos que ubican a las tecnologías como objeto de estudio dan cuenta de las relaciones que se tejen entre diferentes aspectos de la educación y el uso de tecnologías, por ejemplo, entre las políticas educativas y los programas de dotación de infraestructura tecnológica para las escuelas, o entre el diseño de recursos tecnológicos y las necesidades específicas de las comunidades educativas. También denota la importancia de los equipos multidisciplinares en el diseño de los recursos tecnológicos orientados a la Educación, con el fin de que estas atiendan a criterios de fundamentación pedagógica, didáctica, disciplinar y técnica.

\section{formación de procesos formativos en educación superior.}

Esta subcategoría refiere a los aportes y potencial transformador de la tecnología en los procesos educativos en Educación Superior registrados en los diferentes estudios. Al respecto, las 13 unidades de análisis presentadas en la Tabla 6 se diferencian en dos conjuntos, el primero constituido de seis artículos que refieren a estudiantes universitarios en diferentes áreas (Medina y Ortiz, 2013; Martínez, Susano y Espinosa, 2015; Rueda y Parada, 2016; Fabro, 2017; Carrillo y 
Suárez, 2017; Fabro et al., 2020) y el segundo conjunto conformado por siete textos orientados a la formación de profesores tanto inicial como continuada (Richit, 2014; Carmona-Mesa, Salazar y Villa-Ochoa, 2018; Rosa y Orey, 2019; Romo-Vázquez, Barquero y Bosch, 2019; Carmona-Mesa, Cardona y Castrillón-Yepes, 2020; Zampieri y Javaroni, 2020; Vera, Araujo y Veit 2020).

En el primer conjunto se destacan los estudios de Medina y Ortiz (2013) y Rueda y Parada (2016) orientados a la enseñanza de las matemáticas. En el primer estudio, al incluir las calculadoras graficadoras para la solución de problemas aplicados, se reporta que los estudiantes logran mayor precisión conceptual al interpretar gráficas, analizar tendencias de valores en tablas numéricas y generar relaciones entre las diferentes representaciones en la solución del problema delimitado (Medina y Ortiz, 2013). En el segundo estudio se incluye el uso del GeoGebra para fomentar el razonamiento covariacional. Se resalta que la representación ejecutable del software, a diferencia del lápiz y papel, permite a los estudiantes un tránsito más ameno y con menores dificultades por las acciones mentales (Rueda y Parada, 2016).

En cuanto a las cuatro investigaciones restantes, se identifica un énfasis en procesos virtuales orientados a la inclusión escolar (Carrillo y Suárez, 2017), las ciencias morfológicas (Fabro, 2017; Fabro et al., 2020) y el uso de redes sociales se reporta con potencial al incrementar los desempeños de aprendizaje y evaluación, así como la profundidad, participación e integración de saberes formales en estudiantes universitarios regulares (Martínez, Susano y Espinosa, 2015). Además, se fortalecen las competencias comunicativas en el manejo de la lengua escrita de estudiantes con discapacidad auditiva, por medio de herramientas interactivas a través de internet como audio, imágenes llamativas y entendibles, texto o vocabulario adecuado y video con intérprete en cada uno de los módulos (Carrillo y Suárez, 2017).

En particular, las investigaciones en el marco de las ciencias morfológicas diseñan e implementan entornos B-learning (presenciales-vir- tuales), en donde el componente virtual incluye la observación de imágenes, animaciones y videos de sitios web, e-books, foros, aplicaciones de realidad aumentada y modelos histológicos y anatómicos digitales tridimensionales (Fabro, 2017; Fabro et al., 2020). De estas investigaciones se resalta que la utilización de recursos virtuales fomenta aprendizajes significativos, el trabajo colaborativo, la reflexión grupal, la autonomía y el pensamiento cítrico (Fabro, 2017); además, los alumnos destacan la superación de las barreras espaciotemporales de acceso al conocimiento (Fabro et al., 2020).

Por otra parte, los documentos referidos a la formación tanto inicial como continuada de profesores se agrupan en experiencias al interior de cursos específicos (Carmona-Mesa, Salazar y Villa-Ochoa, 2018; Carmona-Mesa, Cardona y Castrillón-Yepes, 2020) y formación en entornos virtuales (Romo-Vázquez, Barquero y Bosch, 2019; Rosa y Orey, 2019; Zampieri y Javaroni, 2020; Vera, Araujo y Veit, 2020). En cuanto a los primeros, Carmona-Mesa, Salazar y Villa-Ochoa (2018), a partir del análisis del primer (uso de calculadoras simples) y último (uso de videojuegos) eje temático de un curso especifico, informan que los profesores se relacionaron con aspectos técnicos de forma fluida e identifican una transformación de un uso personal de la tecnología a un uso educativo. De forma similar, Carmona-Mesa, Cardona y Castrillón-Yepes (2020) resaltan que la tecnología permitió que futuros profesores potenciaran interpretaciones matemáticas de un fenómeno físico, a partir de las diferentes representaciones favorecidas por un simulador computacional.

Respecto a la formación en entornos virtuales a través de tecnologías como Moodle, se destaca el potencial para la difusión, adaptación y formación de profesores en nuevas propuestas educativas como la modelación matemática y para reflexionar críticamente sobre el papel de las matemáticas en la sociedad (Romo-Vázquez, Barquero y Bosch, 2019; Rosa y Orey, 2019); de igual forma, el desarrollo de actividades interdisciplinares que integran tecnologías como Scratch y fomentan el desarrollo del pensamiento computacional (Zampieri y Javaroni, 2020). Además, investigadores como Vera, Araujo y Veit 
(2020) reportan que los entornos virtuales propician la interacción continua entre los diferentes participantes de una comunidad de práctica, aportando a la conformación de nuevas redes de cooperación fundamentadas en los intereses de los participantes. Por último, Richit (2014) reporta como clave la alfabetización digital de profesores para favorecer la integración de la internet en los procesos educativos de manera eficiente y consciente.
El presente estudio se propuso desarrollar un análisis de las tendencias que se reportaron en la revista Uni-pluriversidad entre el 2010 y 2020 , en cuanto a tipos de tecnologías y transformaciones educativas derivadas de integrar tecnologías en la Educación Superior. Lo anterior permitió evidenciar alcances y limitaciones en los modos y patrones de relacionamiento entre los sujetos, el conocimiento y la infraestructura tecnológica privilegiada en las diferentes investigaciones.

Al respecto, resulta importante destacar inicialmente los alcances de las inferencias derivadas de las investigaciones publicadas en la revista. Se registran estudios que reportan evidencia empírica en países como Colombia, Brasil, Argentina, Venezuela, México, España y Portugal (ver Tabla 3); sin embargo, la influencia y proyección que tiene Uni-pluriversidad en la temática es principalmente a nivel de América Latina. En ese sentido, es factible afirmar que la revista brinda cierto grado de representatividad de las dinámicas en la Educación Superior de la región, en cuanto a las formas en que se están adaptando y los retos que se afrontan en la sociedad actual (Jensen, 2019).

En cuanto a la tendencia en los tipos de tecnologías implementadas en educación superior, se destaca la diversidad de alternativas consideradas en las investigaciones y que van desde calculadoras (Medina y Ortiz 2013; Carmona-Mesa, Salazar y Villa-Ochoa, 2018), software educativos como GeoGebra y simuladores (Rueda y Parada, 2016; Carmona-Mesa, Cardona y Castrillón-Yepes 2020) hasta tecnologías como la realidad virtual y aumentada, modelos 3D (Zapata, Marín y Vélez, 2012; Fabro et al., 2020) y lenguajes de programación (Zampieri y Javaroni, 2020). La tendencia en los tipos de tecnología da cuenta de un interés marcado por incorporación de tecnologías soportadas princi-

\section{Discusión y conclusiones}

palmente en la internet y, en menor medida, en los dispositivos móviles.

La tendencia en los tipos de tecnología en la región también pone en evidencia algunos desafíos en cuanto al dinamismo productivo, económico y social actual en América Latina (Finquelievich, 2020), es decir, existe una necesidad de ampliar en investigaciones relacionadas con los dispositivos móviles que toman relevancia en las discusiones contemporáneas. En esa línea, es posible afirmar que las conclusiones derivadas de este estudio se constituyen como insumos para comprender mejor los desafíos en modos y patrones de relacionamiento entre los sujetos y la infraestructura que se experimenta en la región.

Las investigaciones relacionadas con la transformación de procesos formativos en Educación Superior develan poca diversidad en las disciplinas (seis unidades de análisis), y un notorio énfasis en la formación de profesores (trece unidades de análisis). Este aspecto puede ser consecuencia de las particularidades de una revista adscrita a una Facultad de Educación, razón por la cual presenta un énfasis implícito en dicha línea de investigación. Si bien lo anterior no es un aspecto negativo, se constituye como una oportunidad para ampliar la diversidad de estudios que son publicados en la revista de manera que integre, por ejemplo, áreas como ingeniería que permitan fortalecer la tecnología como objeto de estudio.

De igual forma, la transformación de procesos formativos registrada en los estudios refleja un énfasis marcado en evidencia empírica que permite ampliar los modos y patrones de relacionamiento al darle un lugar protagónico al conocimiento en relación con los sujetos y la infraestructura (Ávila-Fajardo y Riascos-Erazo, 2011). Por ejemplo, se identifica una tendencia en las discusiones hacia temas de política pública rel- 
acionados con el fomento de la tecnología en los procesos educativos (Richit, 2014; Martins, 2014; Hugo et al., 2014; Vásquez, 2014). Este aspecto converge con investigaciones como la de Carmona-Mesa, Krugel y Villa-Ochoa (2020) y Teresa y Ithurburu (2020), que intentan ampliar las discusiones sobre las reformas en políticas educativas relacionadas con la integración de tecnología en la región. En particular, Carmona-Mesa, Krugel y Villa-Ochoa (2021) plantean desafíos y alternativas en el caso de la formación de profesores; por su parte, Teresa y Ithurburu (2020) convocan a centrar los argumentos en las desigualdades educativas y las competencias digitales como uno de los problemas ineludibles que enfrenta América Latina en la actualidad. Lo anterior evidencia como la revista amplía las discusiones más allá de los aspectos de capacidad instalada.

Por último, se destaca la tendencia marcada hacia investigaciones cualitativas, solo una publicación cuantitativa y otra mixta. Esto denota una oportunidad para la investigación en la línea de integración de tecnología en los procesos educativos y, en general, para las diferentes líneas de investigación que abordan este objeto de estudio, para la consolidación de trabajos de investigación desde diferentes paradigmas de investigación que apunten a la comprensión sobre las dimensiones, retos y posibilidades de este tipo de tecnologías en la educación superior.

\section{Agradecimientos}

Para los autores del artículo es un gran placer participar con la presente contribución en el aniversario de la revista Uni-pluriversidad, por lo cual se agradece al equipo editorial por tan generosa invitación.

Referencias

Ávila-Fajardo, G. P., \& Riascos-Erazo, S. C. (2011). Propuesta para la medición del impacto de las TIC en la enseñanza universitaria. Educación y Educadores, 14(1), 169-188. doi: 10.5294/edu.2011.14.1.9

Bolívar, W. (2013). El uso de medios y TIC para un humanismo digital. Entrevista a Octavio Henao Álvarez. Uni-pluriversidad, 13(2), 114-118.

Carmona-Mesa, J. A., Cardona Zapata, M. E., y Castrillón-Yepes, A. (2020). Estudio de fenómenos físicos en la formación inicial de profesores de Matemáticas. Una experiencia con enfoque STEM. Uni-pluriversidad, 20(1), e2020101. doi: 10.17533/udea.unipluri.20.1.02

Carmona-Mesa, J. A., Krugel, J., y Villa-Ochoa, J. A. (2021). La formación de futuros profesores en tecnología. Aportes al debate actual sobre los Programas de Licenciatura en Colombia. En A. Richit \& H. Oliveira (Eds.), Formação de professores e tecnologias digitais. Brazil: Livraria da Física.

Carmona-Mesa, J. A., Salazar, J. V. F., y Villa-Ochoa, J. A. (2018). Uso de calculadoras simples y videojuegos en un curso de formación de profesores. Uni-pluriversidad, 18(1), 13-24. doi: 10.17533/udea.unipluri.18.1.02

Carrillo, J., y Suárez, M. S. (2017). Diseño de una estrategia didáctica para cualificar las habilidades escritas en estudiantes universitarios con discapacidad auditiva a través de la web en instituciones de educación superior. Uni-pluriversidad, 17(1), 72-76.

Fabro, A. P. (2017). Los recursos tecnológicos virtuales como favorecedores de la enseñanza y los aprendizajes de ciencias morfológicas. Uni-pluriversidad, 17(2), 81-87. doi: 10.17533/udea.unipluri.17.1.08

Fabro, A. P., Aró, C., Villafañe, N., y Degrave, V. (2020). Integración de las TIC para la enseñanza de las Ciencias Morfológicas en la Universidad: principales aplicaciones utilizadas y valoraciones de docentes y alumnos. Uni-pluriversidad, 20(1), e2020103. doi: 10.17533/udea.unipluri.20.1.04

Finquelievich, S. (2020). América Latina: entre el envejecimiento y el tsunami tecnológico Robótica, inteligencia artificial y trabajo. En A. Rivoir (Ed.), Tecnologías digitales y transformaciones sociales. Desigualdades y 
desafíos en el contexto latinoamericano actual (pp. 15-31). Ciudad Autónoma de Buenos Aires: CLACSO; Montevideo: Facultad de Ciencias Sociales de la Universidad de la República - Uruguay.

Hoyos, C. (2000). Un modelo para investigación documental: guía teórico-práctica sobre construcción de Estados del Arte con importantes reflexiones sobre la investigación. Señal Editora.

Hugo, D., Olavegogeasoechea, M., Orlandini, L., Salica, M. y Ávila, S. (2014). Investigar e innovar la formación CTS inicial de profesores de ciencias aplicando una secuencia de enseñanza-aprendizaje sobre las decisiones tecnológicas. Uni-pluriversidad, 14(2), 72-80.

Jensen, T. (2019). Higher Education in the Digital Era. The current state of transformation around the world. International Association of Universities (IAU).

Marín, V. I., Duart, J. M., Galvis, A. H., y Zawacki-Richter, O. (2018). Thematic analysis of the international journal of educational Technology in Higher Education (ETHE) between 2004 and 2017. International Journal of Educational Technology in Higher Education, 15(1). doi: 10.1186/s41239-018-0089-y

Martínez, M., Susano, J., y Espinosa, J. (2015). Redes sociales y tics en la cátedra universitaria. Uni-pluriversidad, 15(1), 87-99.

Martins, I. P. (2014). Políticas públicas e formação de professores em educação CTS. Uni-pluriversidad, 14(2), $50-62$.

Medina, J. y Ortiz, J. O. (2013). Competencias matemáticas y uso de calculadora gráfica en un contexto de resolución de problemas aplicados. Uni-pluriversidad, 13(3), 14-28.

Muchenski J. C., Miquelin A. F., \& da Silva S. de C. R. (2017). A utilização leiga dos smartphones versus a sua utilização especializada no ambiente escolar. Uni-pluriversidad, 17(1), 25-38.

Ospina, C. (2011). La constitución de identidades reales en el marco virtual de las redes tecnológicas. Uni-pluriversidad, 11(3), 35-42.

Quiroga-Parra, D. J., Torrent-Sellens, J., Patricia, C., y Zorrilla, M. (2017). Usos de las TIC en América Latina. Revista chilena de ingeniería, 25(2), 289-305.

Quiroz-Vallejo, D. A. (2020). Computational Thinking, Curriculum and Interdisciplinarity. An interview with Johannes Krugel. Uni-pluriversidad, 20(1), e2020110-e2020110.

Ramírez, E. (2011). Simbiosis de una Combinación entre la Aplicación de un Prototipo Virtual-CDROM Educativo-y una Plataforma Virtual-Campus Virtual GITT-para la Construcción y Gestión del Conocimiento. Uni-pluriversidad, 11(1), 54-68.

Richit, A. (2014). Formação de Professores em Tecnologias Digitais: desdobramentos nas práticas escolares em face do Programa Um Computador por Aluno. Uni-pluriversidad, 14(3), 81-93.

Romo-Vázquez, A., Barquero, B., y Bosch, M. (2019). El desarrollo profesional online de profesores de matemáticas en activo: una unidad de aprendizaje sobre la enseñanza de la modelización matemática. Uni-pluriversidad, 19(2), 161-183. doi: 10.17533/udea.unipluri.19.2.09

Rosa, M., y Orey, D. C. (2019). Mathematical modelling as a virtual learning environment for teacher education programs. Uni-pluriversidad, 19(2), 80-102. doi: 10.17533/udea.unipluri.19.2.04

Rueda, N., y Parada, S. (2016). Razonamiento covariacional en situaciones de optimización modeladas por Ambientes de Geometría Dinámica. Uni-pluriversidad, 16(1), 51-63.

Teresa, L., y Ithurburu, V. (2020). Las políticas digitales en educación. Una cuestión de derechos. En A. Rivoir (Ed.), Tecnologías digitales y transformaciones sociales. Desigualdades y desafíos en el contexto latinoamericano actual (pp. 15-31). Ciudad Autónoma de Buenos Aires: CLACSO; Montevideo: Facultad de Ciencias Sociales de la Universidad de la República - Uruguay.

Vargas, C. (2011). El Gobierno Electrónico o e-Gobierno. Uni-pluriversidad, 11(1), 69-84.

Vásquez, Á. (2014). Enseñanza, Aprendizaje y Evaluación en la Formación de Docentes en Educación CTS en el contexto del siglo XXI. Uni-pluriversidad, 14(2), 37-49. 
Velasco, C., y Gómez, P. (2019). Calidad y dificultad de los cuestionarios de un MOOC. Unip/uriversidad, 19(2), 124-143. doi: 10.17533/udea.unipluri.19.2.06

Vera Rey, E. A., Araujo, I., y Veit, E. (2020). Acciones para el cultivo de una Comunidad de Práctica de profesores de Física: análisis de los procesos de participación periférica legítima a través de un curso virtual sobre Recursos Educativos Abiertos para la enseñanza de la Física. Uni-pluriversidad, 20(1), e2020107. doi: 10.17533/udea.unipluri.20.1.08

Villa-Ochoa, J. A., y Castrillon-Yepes, A. (2020). Temas y tendencias de investigación en América Latina a la luz del pensamiento computacional en Educación Superior. In G. Toledo Lara (Ed.), Políticas, Universidad e innovación: retos y perspectivas (pp. 235-248). J.M Bosch. doi: 10.2307/j.ctv1dv0v33.14

Zampieri, M. T., y Javaroni, S. L. (2020). A Dialogue Between Computational Thinking and Interdisciplinarity using Scratch Software. Uni-pluriversidad, 20(1), e2020105. doi: 10.17533/udea.unipluri.20.1.06

Zapata, D., Marín, A., y Vélez, Y. (2012). Metodología de producción de diseño gráfico para un entorno de enseñanza y aprendizaje en un mundo virtual tridimensional (mv3d). Uni-pluriversidad, 12 (1), 14-24. 